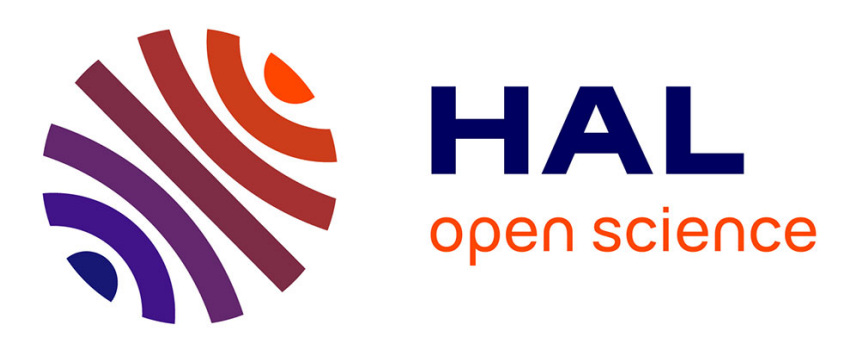

\title{
Dynamiques funéraires et faciès culturels. L'exemple du sud-ouest de la France et du Nord de l'Espagne dans la première moitié du Ier millénaire avant notre ère
}

Stéphanie Adroit

\section{- To cite this version:}

Stéphanie Adroit. Dynamiques funéraires et faciès culturels. L'exemple du sud-ouest de la France et du Nord de l'Espagne dans la première moitié du Ier millénaire avant notre ère. Archimède: archéologie et histoire ancienne, 2014, 1, pp.203-216. hal-01967498

\section{HAL Id: hal-01967498 \\ https://hal.science/hal-01967498}

Submitted on 31 Dec 2018

HAL is a multi-disciplinary open access archive for the deposit and dissemination of scientific research documents, whether they are published or not. The documents may come from teaching and research institutions in France or abroad, or from public or private research centers.
L'archive ouverte pluridisciplinaire HAL, est destinée au dépôt et à la diffusion de documents scientifiques de niveau recherche, publiés ou non, émanant des établissements d'enseignement et de recherche français ou étrangers, des laboratoires publics ou privés. 


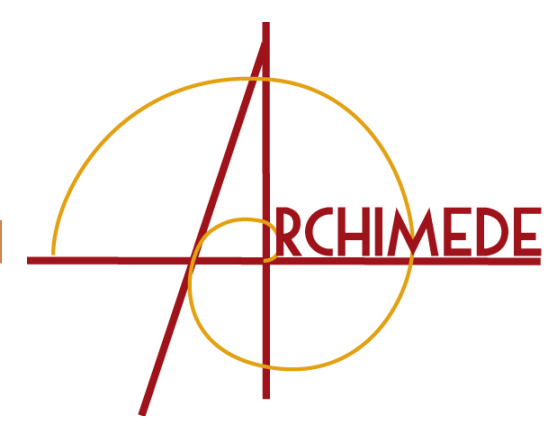

\title{
DYNAMIQUES FUNÉRAIRES ET FACIĖS CULTURELS \\ L'EXEMPLE DU SUD-OUEST DE LA FRANCE ET DU NORD DE L'ESPAGNE DE LA PREMIÈRE MOITIÉ DU ler MILLÉNAIRE AVANT NOTRE ĖRE
}

\author{
Stéphanie ADROIT \\ Doctorante, \\ Casa de Velázquez, \\ Université de Toulouse II, \\ TRACES (UMR 5608). \\ stephanie.adroit@orange.fr
}

\section{RÉSUMÉ}

Dans le cadre spatio-temporel que nous avons choisi de présenter ici, les archéologues ont souvent mis en exergue les similitudes existantes, sur la base du mobilier, entre les deux versants des Pyrénées. Ce constat nous a donc amené à nous interroger sur les liens éventuels décelables - au-delà des objets - dans les gestes et les comportements funéraires de ces communautés, afin d'identifier les possibles éléments qui les rapprochent ou les différencient d'un point de vue des idées symboliques, des mécanismes sociaux, économiques et culturels. C'est dans cette perspective d'évaluer les dynamiques des pratiques funéraires que nous avons recours à une chronologie dilatée afin de nous interroger sur la variabilité des pratiques funéraires selon les périodes concernées ainsi que dans pratiques funéraires, Bronze final, premier âge du Fer, sud-ouest de la France, nord de l'Espagne.
Los arqueólogos, basándose en los ajuares funerarios, han señalado a menudo las semejanzas que se dan entre las dos vertientes de los Pirineos durante el marco espacio-temporal que hemos elegido investigar. Este cotejo nos induce a interrogarnos sobre la relación que, más allá de los objetos, podría reflejarse en los gestos y comportamientos asociados a las prácticas funerarias de las comunidades implicadas. De esta manera, podríamos identificar los elementos que las acercan o las diferencian desde la perspectiva de las ideas simbólicas y de los mecanismos sociales, económicos y culturales. Con el fin de evaluar las dinámicas de las prácticas funerarias, recurriremos a una cronología dilatada que nos permita calibrar las variantes de éstas en periodos concretos, así como identificar dinámicas más amplias, de alcance supra-regional.
Palabras clave Necrópolis de cremación, prácticas funerarias, Bronce final, primera edad del Hierro, suroeste de Francia norte de la Península Ibérica. 


\section{EN GUISE DE PROLOGUE}

D'entrée de jeu je souhaiterais souligner que cet article entend présenter des recherches doctorales en cours ce qui, par voie de conséquence, en fait plutôt un exposé méthodologique qu'une analyse de synthèse. La genèse de mon sujet de thèse découle du constat dressé par plusieurs protohistoriens - sur la base de la circulation et de I'utilisation des objets (ex. : les accessoires vestimentaires et les parures corporelles [1], les armes [2]) des interactions fortes qui existaient entre les deux versants du massif des Pyrénées à l'âge du Fer [3]. Cette constatation m'a donc amené à m'interroger sur les liens éventuels existants, au-delà des objets, dans les gestes et les comportements funéraires des communautés situées de part et d'autre des Pyrénées. Dans la littérature archéologique, les pratiques funéraires sont souvent utilisées dans la caractérisation des groupes culturels archéologiques, sur le principe quasi universel selon lequel chaque population utilise face à la « Mort » tout un ensemble de pratiques et de gestes qui lui est propre [4]. Le domaine pyrénéen et ses marges s'inscrit dans un contexte marqué par l'apparition, entre le Bronze final is et le Bronze final iıa, puis la généralisation rapide de funérailles caractérisées par la crémation du corps des défunts sur une aire de crémation. Dans l'espace géographique et chronologique que je me suis fixé pour étude, les pratiques funéraires occupent une place de premier rang dans la définition des groupes culturels.

[1] Les liens unissant le Languedoc-Roussillon et le nord-est de la péninsule Ibérique à l'âge du Fer ont conduit les archéologues à parler de faciès ibéro-languedocien (GAILlEDRAT 1997). Les relations qui unissent la Meseta et l'Aquitaine sont uniquement basées sur des similitudes observées au niveau des artefacts: les fibules en alliage cuivreux dites de type navarro-aquitain, les agrafes de ceinture en alliage cuivreux à plusieurs crochets (CERdeño 1978 ; Cendeño \& CHORDÁ 2004).

[2] L'épée de Larresingle dans le Gers se rapproche des épées du type II (groupe II) de F. Quesada, les épées à antennes d'Aguilar de Anguita et du bassin d'Arcachon sont d'un type identique.

[3] EsCudÉ-Quillet 1998.

[4] Il faut exclure de cette généralisation les gisements funéraires dits « de catastrophe » (si tant est qu'ils en soient) qui relèvent d'une crise de mortalité : épidémies, guerres, accidents naturels, etc.

Figure 1 : principaux groupes culturels identifiés entre Garonne et Èbre. 1. D’après Mohen \& Coffyn 1970, fig. 10 , p. 132 ;

2. Garcia 2004, p. 28 ; 3. Royo Guillén 2000, fig. 1, p. 42 et 4. López Cachero \&. Rovira i Hortalà 2012, fig. 1, p. 38.
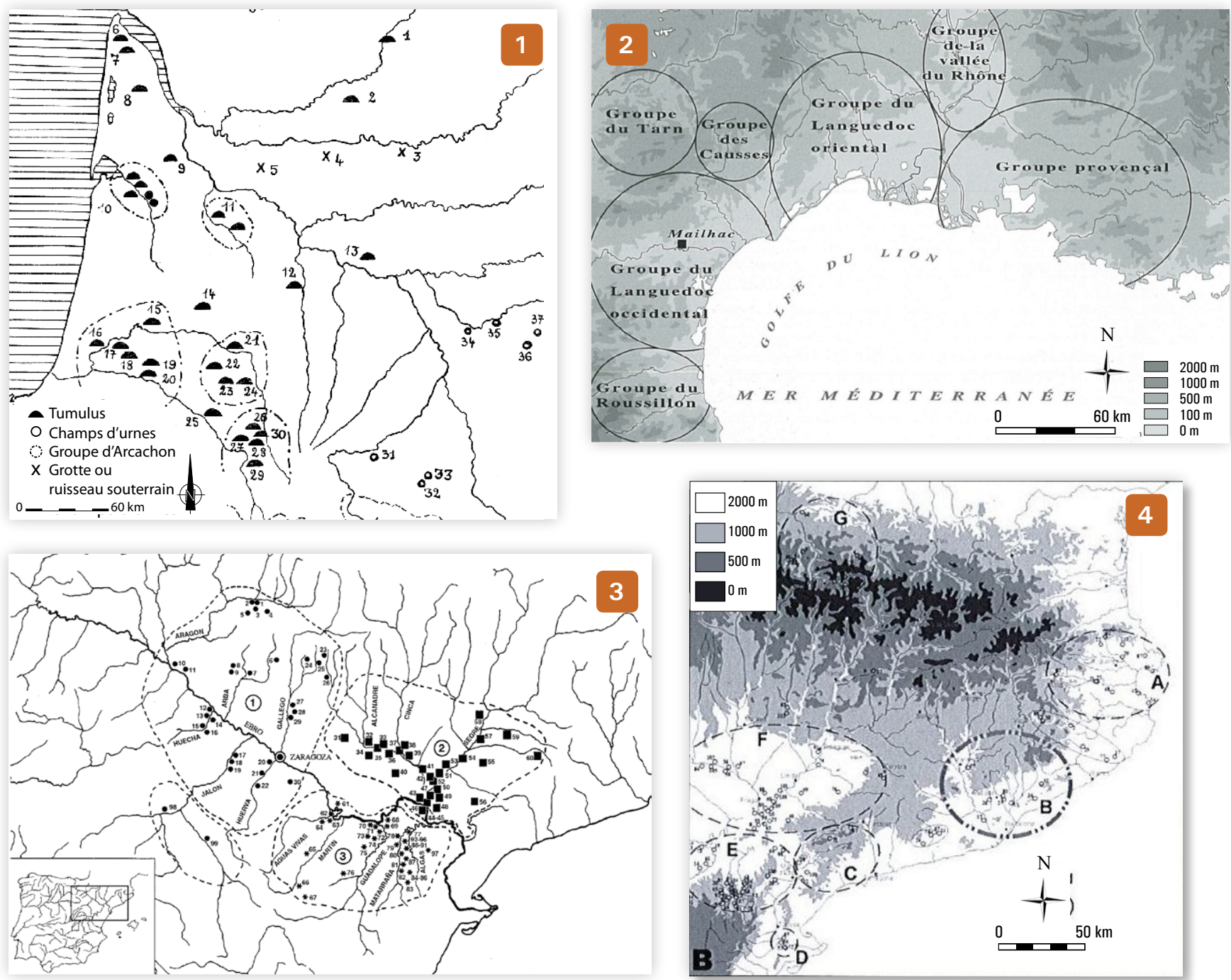
En effet, plusieurs espaces protohistoriques ne sont bien documentés, en l'état actuel des connaissances, que par des données archéologiques issues de la sphère funéraire, d'où le rôle déterminant des pratiques funéraires dans la définition de ces groupes et des faciès culturels des sociétés protohistoriques [5] (Fig. 1). Au cours de I'histoire de la discipline, les pratiques funéraires ou seulement un aspect de la « chaîne opératoire » des funérailles - tel que l'usage de la crémation du cadavre par opposition à celui de l'inhumation, l'antagonisme supposé des structures en fosse et des tumulus - ont été sollicitées, à vrai dire souvent de manière excessive ou réductrice, dans la définition des cultures archéologiques européennes de l'âge du Fer : en témoignent dans la langue des expressions telles que « civilisation des Champs d'Urnes » ou «culture des Tumulus » [6]. Or nous sommes conscients aujourd'hui que l'érosion naturelle et/ou anthropique abîment les superstructures funéraires, ce qui biaise notre vision architecturale des structures si nous évacuons prématurément les phénomènes taphonomiques du discours [7]. De surcroît, les pratiques funéraires doivent être étudiées dans l'intégralité de leur processus sinon nous encourons le risque d'effacer les spécificités funéraires des complexes sépulcraux en isolant artificiellement les éléments. Les cartes qui en découlent ne reflètent donc pas de manière objective le panorama des pratiques funéraires du passé.

\section{PAR DELÀ LES OBJETS DANS LA TOMBE}

Pour élaborer une grille d'analyse des nécropoles à crémation, je me suis d'abord inspirée des travaux menés par J.-M. Luce sur les pratiques funéraires de l'âge du Fer en Grèce [8]. Il tente de montrer qu'à des époques précises, dans certaines zones de la Grèce, les pratiques funéraires ont été des géosymboles dans le sens dans lequel I'entendait J. Bonnemaison. Il explique que pour une étape chronologique donnée, ce n'est pas la présence de tel ou tel trait funéraire qui définit une nécropole mais leur combinaison dans un processus ; c'est ce qu'il appelle la « séquence funéraire ». Cette combinaison peut être spécifique à la communauté qui la met en place progressivement dans le temps, en suivant souvent des phases distinctes [9]. Bien évidemment une « séquence

[5] Ce constat vaut surtout pour la région administrative de l'Aquitaine où les informations concernant les habitats protohistoriques sont largement déficitaires par rapport aux gisements funéraires. Cet état de fait découle simplement de l'intérêt précoce des érudits locaux pour les nécropoles tumulaires aux dépens des habitats contemporains. Ces déséquilibres, grâce au développement de l'archéologie préventive, se sont amenuisés mais les données publiées sur l'habitat restent ténues.

[6] Bosch-Gimpera 1932 ; Louis et al. 1960 ; JANin 1994 ; LóPEZ Cachero 2005.

[7] De même l'érosion des superstructures funéraires est préjudiciable à l'étude des pratiques commémoratives qui peuvent intervenir au funéraire » peut caractériser une ou plusieurs nécropoles et ainsi correspondre, soit à un espace homogène géographiquement, soit discontinu. En évacuant volontairement de l'étude le mobilier spécifiquement associé aux défunts [10], nous privilégions ce qui relève des représentations collectives de la mort et de l'identité culturelle plutôt que les identités individuelles des défunts ou les choix particuliers opérés par leurs proches. Selon J.-M. Luce le mobilier funéraire déposé dans une tombe grecque se rapporte d'abord à l'identité personnelle du défunt (âge au décès, genre, statut social, activités exercées au cours de sa vie) alors que les modes de traitement du cadavre, l'architecture funéraire qui en découle et la gestion des espaces sépulcraux répondent à un nombre d'options plus limité et sont des éléments porteurs de pratiques partagées par l'ensemble de la communauté qui les admet comme normales ou non [11]. A contrario le mobilier est plutôt choisi pour l'individu ; de fait, il comporte souvent des objets personnels qui appartenaient au défunt. Néanmoins, dans les contextes chrono-culturels étudiés, nous pensons qu'il est possible aussi d'extraire des informations à partir du mobilier d'accompagnement qui peuvent révéler des phénomènes culturels récurrents ainsi que des changements à l'échelle de la communauté dans la composition des dépôts au fil du temps : quantité de vases déposés dans les tombes, répartition quantitative des différentes catégories fonctionnelles, position du mobilier d'accompagnement, traces de chauffe sur le mobilier métallique indiquant un éventuel passage sur le bûcher funéraire avec le défunt, etc. En effet, la présence dans les tombes de certaines catégories fonctionnelles pourrait correspondre à un espace géographique précis et donc obéir à des contraintes culturelles. De même que la quantité et la forme des vases déposés dans les tombes peuvent découler de normes très différentes concernant les habitudes de consommation alimentaire. Par ailleurs, il ne faut pas exclure le fait que l'identité personnelle du défunt puisse être symbolisée dans d'autres champs que celui du strict mobilier funéraire, comme dans le choix du rite, le type de tombe ou à travers d'autres éléments architecturaux (ex : stèle anthropomorphe). Les recherches de L. Baray sur les pratiques funéraires du Bassin parisien défendent également l'idée que les pratiques funéraires sont porteuses d'une « identité collective » [12].

moment de la fermeture de la tombe, ou après elle, les vestiges qui en témoignent se trouvent en principe à la surface du sol.

[8] LUCE 2007.

[9] Ibid., p. 44.

[10] Le fait de ne pas mettre l'étude du mobilier funéraire stricto sensu au centre d'une analyse sur les pratiques funéraires se justifie dans la mesure où les contextes de découverte de quelques objets sont incertains voire inconnus.

[11] LUCE 2007, p. 40-42.

[12] BARAY 2003. 
D'après lui, les pratiques funéraires expriment des choix qui sont fixés par la communauté qui les admet comme normales ou non, c'est-à-dire « qu'à travers les pratiques funéraires, les sociétés affichent une partie de leur système social et culturel (I'uniformité) et l'individu "exprime ses particularités" (différenciations), sur le mode de la variation, qui seule autorise une vision dynamique de l'évolution historique » [13]. Ainsi, pour L. Baray les variations répertoriées dans les rituels funéraires sont des différences à la fois de degré et de nature. Les variations de degré sont appréciées sur la base de la présence d'un élément qui peut être attestée dans plusieurs régions et qui varie en fonction d'un pourcentage alors que les variations de nature permettent de faire une distinction selon un schéma classique de présence/ absence. D'après lui, les pratiques funéraires uniformes ou dont la forme varie dans un registre limité expriment les représentations collectives. Au contraire, les pratiques funéraires dont la forme est liée à des contingences variées symbolisent l'identité individuelle.

L'objectif majeur de ce mémoire de thèse consiste à comparer, à partir d'une large gamme de critères (monuments funéraires, gestion des espaces sépulcraux, mode de dépôt des restes humains brûlés, etc.) les séquences funéraires de plusieurs nécropoles/tumulus pour une étape chronologique donnée. Néanmoins, plusieurs obstacles ont conditionné le développement d'une typologie de ces critères, d'abord au niveau de la nécropole, ensuite à l'échelle de la tombe. Les nécropoles et leurs tombes rassemblent en elles-mêmes tout un panel d'informations que l'archéologue se doit d'observer rigoureusement. Cependant, dans la réalité, nous savons que les fouilles des sites que nous étudions n'ont pas fait I'objet d'une rigueur scientifique analogue - pour s'en convaincre il suffit de lire les carnets de fouilles de la fin $d u x x^{e}$ siècle et de la première moitié $d u x x^{e}$ siècle - ce qui tend à biaiser toute tentative d'analyse comparative des pratiques funéraires. Ainsi, il s'avère illusoire de vouloir décrire dans une typologie stricte et précise toutes les composantes des chaînes opératoires des pratiques funéraires. Certaines observations archéologiques qui nous paraissent être significatives pour comprendre les choix opérés ou proposés par la communauté dans le processus funéraire n'ont pas été documentées dans tous les espaces funéraires étudiés. Par exemple, les études anthropologiques sur les restes humains brûlés déposés dans les tombes sont très inégales en fonction des régions, parfois même absentes dans certaines zones où les fouilles de nécropoles à crémation sont pourtant anciennes [14]. De surcroît, en raison de la quantité des données disponibles mais également de leur qualité très disparate, notre travail ne prétend pas à l'exhaustivité.
Il prend appui sur une sélection raisonnée de sites répartis tant soit peu de manière homogène dans l'espace et dans le temps.

Suite à l'établissement de la « séquence funéraire » spécifique à un site pour une étape chronologique concrète, il nous faut nous interroger sur cette spécificité. Est-ce que la « séquence funéraire » est partagée, c'est-à-dire estelle commune à d'autres sites ? Dans l'affirmative, quelle est la répartition géographique de ces sites qui présentent une «séquence funéraire » identique ? Les séquences communes à plusieurs nécropoles apparaissent-elles dans un espace homogène et restreint, ou bien sur des surfaces importantes et discontinues car partagées par des nécropoles présentant des séquences autres ? La répartition géographique de ces séquences est-elle conditionnée par des déterminismes d'ordre géographique ? Par là même, il est intéressant de s'interroger sur cette spécificité d'un point de vue chronologique. Ces « séquences funéraires » se sont-elles maintenues ou se sont-elles modifiées dans le temps ? L'étude sur la longue durée permet de saisir justement les dynamiques funéraires, les moments de rupture, de transition. In fine, l'objectif principal de cette étude comparative, de part et d'autre des Pyrénées, est de réaliser une cartographie des séquences funéraires du Bronze final et du premier âge du Fer afin d'observer les éléments qui rapprochent ou au contraire distinguent les communautés du nord de la péninsule Ibérique et du sud-ouest de la France. Dans un second temps, on pourra comparer ces cartes à d'autres cartes de répartition afin de s'interroger sur les liens et les discordances avec d'autres traits des cultures matérielles supposés entrer dans la définition des groupes et des faciès culturels précis.

\section{LES «CHAÎNES OPÉRATOIRES » DES PRATIQUES FUNÉRAIRES}

Pour ce faire, il faut prendre en compte toutes les étapes des pratiques funéraires identifiables archéologiquement au sein d'un site, depuis la crémation ou I'inhumation du corps du défunt en passant par la mise en terre jusqu'aux rites funèbres ou commémoratifs sur la tombe, en établissant des critères précis mais pas immodérés afin de ne pas créer des groupes artificiels qui seraient le reflet de groupes résultant de fouilles récentes et d'autres de fouilles anciennes. Il s'agit de suivre une véritable «chaîne opératoire » des pratiques funéraires dont l'ensemble des critères sert à la mise en place d'une typologie funéraire (Fig. 2).

[13] Ibid., p. 345.

[14] Duday et al. 2000. 


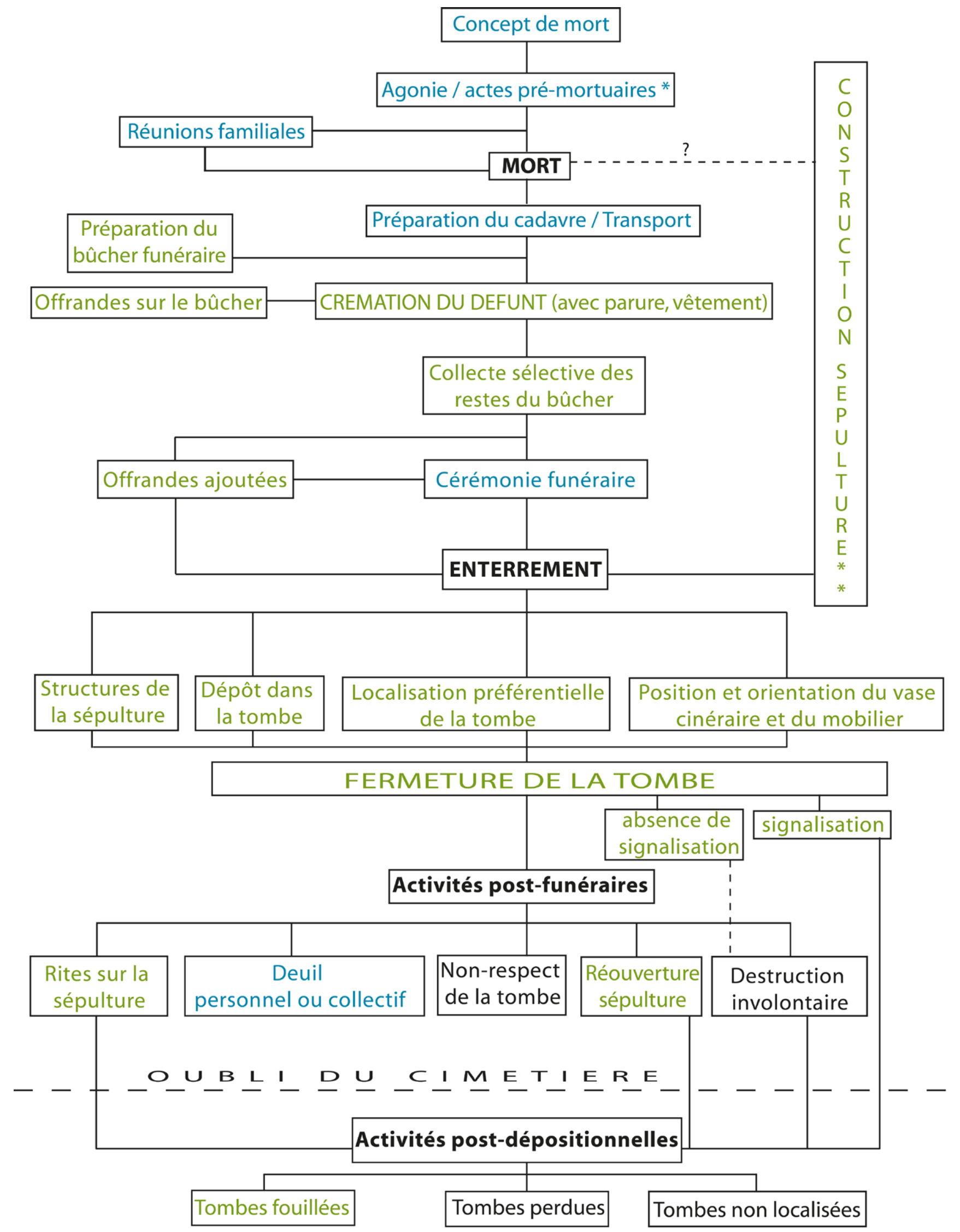

* Cette étape du rituel funéraire n'est pas présente dans tous les cas (mort violente).

** Il est difficile de dire à quel moment précis la tombe est construite. La préparation de cette dernière peut commencer à la mort de l'individu ou bien plus tard dans le rituel, parrallèlement à d'autres activités. 
Pour commencer, j'ai distingué le type de gisement auquel se rapporte la nécropole en différenciant notamment trois niveaux : s'agit-il de tombe en fosse, de tombe à même le sol, de tombe au-dessus du sol naturel. Par la suite, nous prenons en compte le type de nécropole: Est-ce que les nécropoles adoptent un plan linéaire ou un plan multipolaire ? Est-ce que la disposition des tombes est régulière/homogène ? Nous avons également noté les cas où les sépultures se superposent/se recoupent, ce qui est un indice d'un manque de signalisation ou la conséquence d'une compartimentation familiale qui sous-entend la réutilisation d'un espace déterminé par les défunts d'un même lignage. Toutes les structures parafunéraires répertoriées au sein d'un site sont également prises en compte, comme les ustrina, les dépôts annexes, etc. Ensuite, pour ce qui de l'architecture funéraire externe, j'ai décrit les plans/les types de structures d'entourages, les techniques de construction usitées, la présence éventuelle de mobilier placé dans la masse tumulaire, etc. La totalité des dimensions des structures qui avaient été documentées sur le terrain ont été prises en compte ; à partir de ces mesures nous avons établi des classes d'ordre de grandeur, des superficies, du volume des fosses et des tertres, etc. Par ailleurs, les fosses funéraires ont été précisément décrites mais également la nature de leur comblement (versement des restes du bûcher funéraire dans la tombe, indices d'un espace vide comblé progressivement). Les différents modes de dépôt des restes humains brûlés ont été répertoriés (Fig. 3), de même que les catégories fonctionnelles des artefacts déposés à l'intérieur du vase cinéraire.

\section{TENTATIVE DE CARTOGRAPHIE DES « SÉQUENCES FUNÉRAIRES »}

Pour le moment, les informations ont été recensées dans une base de données mais elles n'ont pas encore été traitées. Cependant, pour illustrer au mieux mon propos et ma démarche méthodologique, je partirai de quelques exemples développés au cours de mon Master 2 qui traitait du même thème. En premier lieu, nous avions divisé la période comprise entre le Bronze final IIIb et le premier âge du Fer en quatre étapes typochronologiques. Après avoir dressé la « séquence funéraire » de chaque gisement funéraire, à partir de la présence/absence d'une soixantaine de critères, nous avons utilisé la méthode des analyses factorielles de correspondance afin de vérifier si l'on observait d'éventuels regroupements de nécropoles (Tableau. 1). Dans un deuxième temps, ces groupes funéraires furent cartographiés. Cette opération fut répétée pour toutes les étapes typo-chronologiques.

\section{ÉTAPE I : les nécropoles du Bronze final IIIB et de l’horizon initial du premier âge du Fer ancien}

Ainsi pour le Bronze final inb et I'horizon ancien de la première étape du premier âge du Fer, nous avions sélectionné 32 sites relativement bien documentés dans le sud-ouest de la France et le nord de la péninsule Ibérique. In fine, pour la première étape trois groupes funéraires ont pu être identifiés (Fig.4).

\section{Groupe funéraire 1}

Le premier élément qui différencie ce groupe des deux autres est lié à la monumentalité des superstructures funéraires qui avoisinent fréquemment les $50 \mathrm{~m}^{2}$ de superficie (le diamètre est compris entre 8 et $18 \mathrm{~m}$ ). Ces tumulus présentent également la particularité d'avoir un cercle de galets (ou plusieurs cercles concentriques) à la base du tertre et de grandes surfaces d'accumulation de galets à l'intérieur de la masse tumulaire qui sont délicates à interpréter ainsi que des pavages de galets jointifs [15].

[15] Mohen 1980, p. 117, fig. 60.

Figure 3 : typologie inspirée de la publication de Baray 2003 (élaboration S. Adroit)
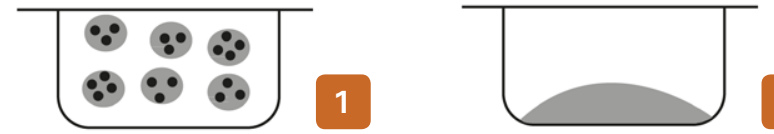

2
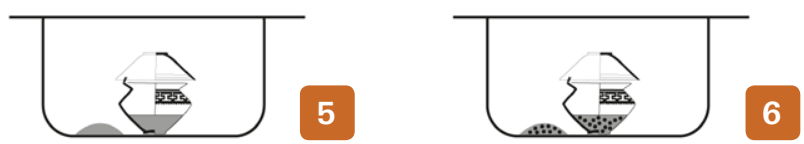

5

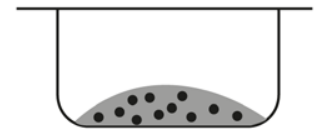

3

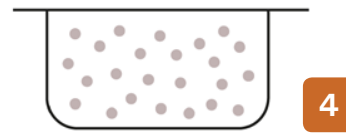

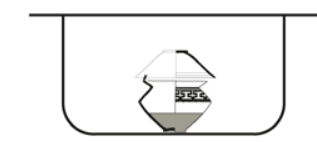

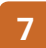

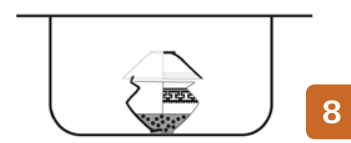

1. Ossements humains brûlés mélangés à un sédiment cendreux/charbonneux dans la fosse ; 2 . Amas d'ossements humains brûlés ; 3. Amas d'ossements humains brûlés mélangés avec un sédiment cendreux/charbonneux ; 4 . Ossements humains brûlés en vrac ; 5 . Ossements humains brûlés à l'intérieur du vase cinéraire et à l'extérieur du contenant; 6 . Ossements humains brûlés et sédiment cendreux/charbonneux à l'intérieur du vase cinéraire et à l'extérieur du contenant ; 7 . Ossements humains brûlés à I'intérieur du vase cinéraire ; 8. Ossements humains brûlés et sédiment cendreux/charbonneux à l'intérieur du vase cinéraire. 
Tableau 1 : tableau des critères permettant de distinguer les différents types de « séquence funéraire " (critères utilisés pour les AFC)

\section{CRITÈRES}

\begin{tabular}{|c|c|}
\hline 1 & Dépôt de crémation primaire. \\
\hline 2 & Dépôt de crémation secondaire. \\
\hline 3 & Bûcher funéraire infra-tumulaire. \\
\hline 4 & Sépulture individuelle. \\
\hline 5 & Sépulture plurielle. \\
\hline 6 & Dépôt de crémation « symbolique ». \\
\hline 7 & Dépôt de crémation " exhaustif ». \\
\hline 8 & Absence de dépôt d'os. \\
\hline 9 & $\begin{array}{l}\text { Dépôt de crémation dans un } \\
\text { contenant (céramique, matière } \\
\text { périssable, coffre, etc.) }\end{array}$ \\
\hline 10 & $\begin{array}{l}\text { Dépôt de crémation hors de } \\
\text { tout contenant conservé. }\end{array}$ \\
\hline 11 & $\begin{array}{l}\text { Sépulture principale en fosse } \\
\text { creusée dans le substrat. }\end{array}$ \\
\hline 12 & $\begin{array}{l}\text { Sépulture principale déposée } \\
\text { au-dessus du sol naturel } \\
\text { à la base du tertre. }\end{array}$ \\
\hline 13 & $\begin{array}{l}\text { Sépulture adventice aménagée } \\
\text { dans le volume tumulaire. }\end{array}$ \\
\hline 14 & $\begin{array}{l}\text { Présence d'un coffre en } \\
\text { pierre, en adobe, etc. }\end{array}$ \\
\hline 15 & $\begin{array}{l}\text { Loculus funéraire comblé avec } \\
\text { les restes (cendres, charbons } \\
\text { de bois) du bûcher funéraire. }\end{array}$ \\
\hline 16 & $\begin{array}{l}\text { Loculus fermé avec une dalle } \\
\text { en pierre ou un système } \\
\text { de fermeture en bois. }\end{array}$ \\
\hline 17 & $\begin{array}{l}\text { Superficie des tertres ou des } \\
\text { enclos comprise entre } 0 \text { et } 10 \mathrm{~m}^{2} \text {. }\end{array}$ \\
\hline & $\begin{array}{l}\text { Superficie des tertres ou des enclos } \\
\text { comprise entre } 10 \text { et } 20 \mathrm{~m}^{2} \text {. }\end{array}$ \\
\hline
\end{tabular}

19 Superficie des tertres ou des enclos comprise entre 20 et $50 \mathrm{~m}^{2}$.

20 Superficie des tertres ou des enclos supérieure à $50 \mathrm{~m}^{2}$.

21 Monuments de forme circulaire / ovale.

22 Monuments de forme quadrangulaire.

23 Structures d'entourages ou enclos larges.

24 Structures d'entourages

24 ou enclos étroits.

25 Fossé d'enclos en périphérie des monuments.

26 Enclos concentriques.

27 Préparation de l'assise du

27 tertre (décapage, dallage)

28 Volume tumulaire constitué d'un amas pierreux en vrac.

29 Volume tumulaire constitué de pierres posées horizontalement.

30 Volume tumulaire de terre et/ou sable.

31 Volume mixte terre et pierres.

32 Amas charbonneux en dehors de

32 l'espace sépulcral stricto sensu.

Réutilisation d'un monument

33 funéraire antérieur au Bronze final III et au premier âge du Fer.

34 Dépôts répétés de sépultures 34 au sein d'un même tumulus.

35 Présence de sépultures adventices

36 Mobilier déposé sur la superstructure de fermeture/couverture de la tombe.
37 Structures pavées au sein du monument funéraire.

38 Tessons non brûlés.

39 Tessons brûlés.

40 Dépôts de faune.

41 Nécropole en situation dominée (plaine).

42 Nécropole en situation dominante (relief).

43 Plan unipolaire.

44 Plan multipolaire (et quelquefois synchronique)

45 Plan linéaire.

46 Disposition (des tombes) aléatoire.

47 Disposition (des tombes) régulière (alignement / espacement).

48 Effectif compris entre 2 et 30 sépultures

49 Effectif compris entre 31

49 et 80 sépultures.

50 Effectif compris entre 81 et 500 sépultures.

51 Effectif supérieur à 500 sépultures.

52 Recoupement/Superposition de tombes.

53 Stèle(s) en pierre ou négatif d'un dispositif de signalisation en bois.

54 Sujet(s) inhumé(s) au sein de la nécropole.
Par ailleurs, lorsqu'il existe plusieurs sépultures au sein d'un même tertre, les sépultures adventices sont souvent aménagées dans le volume tumulaire, ce qui permet parfois d'observer la chronologie relative de dépôt mais aussi de subodorer un mode de signalisation des tombes lorsque les dépôts funéraires ne se recoupent pas. Enfin, contrairement aux deux autres groupes, ces nécropoles tumulaires ne livrent en général qu'une dizaine de structures funéraires alors que les nécropoles présentant des structures en fosse comptabilisent des centaines de sépultures.

\section{Groupe funéraire 2}

Le deuxième groupe partage certains types avec le groupe précédent, comme des structures tumulaires, avec des superficies moindres mais qui sont relativement importantes pour quelques-unes, la présence d'un ou de plusieurs cercles de galets ceinturant les tumulus, ainsi qu'un effectif total compris entre une vingtaine et une centaine de structures funéraires. En revanche, ce groupe introduit également des nouveautés architecturales. En effet, les dépôts funéraires sont, soit déposés dans un loculus sans aucune protection particulière [16], soit placés dans une ciste en pierres [17]. Les superstructures funéraires repérées sont de plusieurs types. Il s'agit en général de petits tertres circulaires qui mesurent entre 1 et $10 \mathrm{~m}$ de diamètre. Ces tumulus sont dotés d'un (ou de plusieurs) cercle(s) de pierres étroit(s) entourant une masse tumulaire fabriquée de façon variée : mélange de terre et de pierres, amas pierreux en vrac, pierres posées à plat, etc. Pour ce qui est de l'organisation interne de ces nécropoles, il n'y a pas vraiment de changement par rapport aux autres groupes. En effet, les structures funéraires peuvent être disposées, soit de manière aléatoire, soit en montrant des phénomènes d'accrétions de tombes autour de noyaux «fondateurs ». L'autre élément à prendre en considération est que ces nécropoles se trouvent à des emplacements topographiques dominants, contrairement à la majorité des nécropoles des autres groupes.

[16] Puig Alt à Roses (Alt Empordà, Girona).

[17] Ayer à Bordes-sur-Lez (Ariège), la Montagne de Benqué (Haute-Garonne), Arihouat à Garin (Haute-Garonne). 


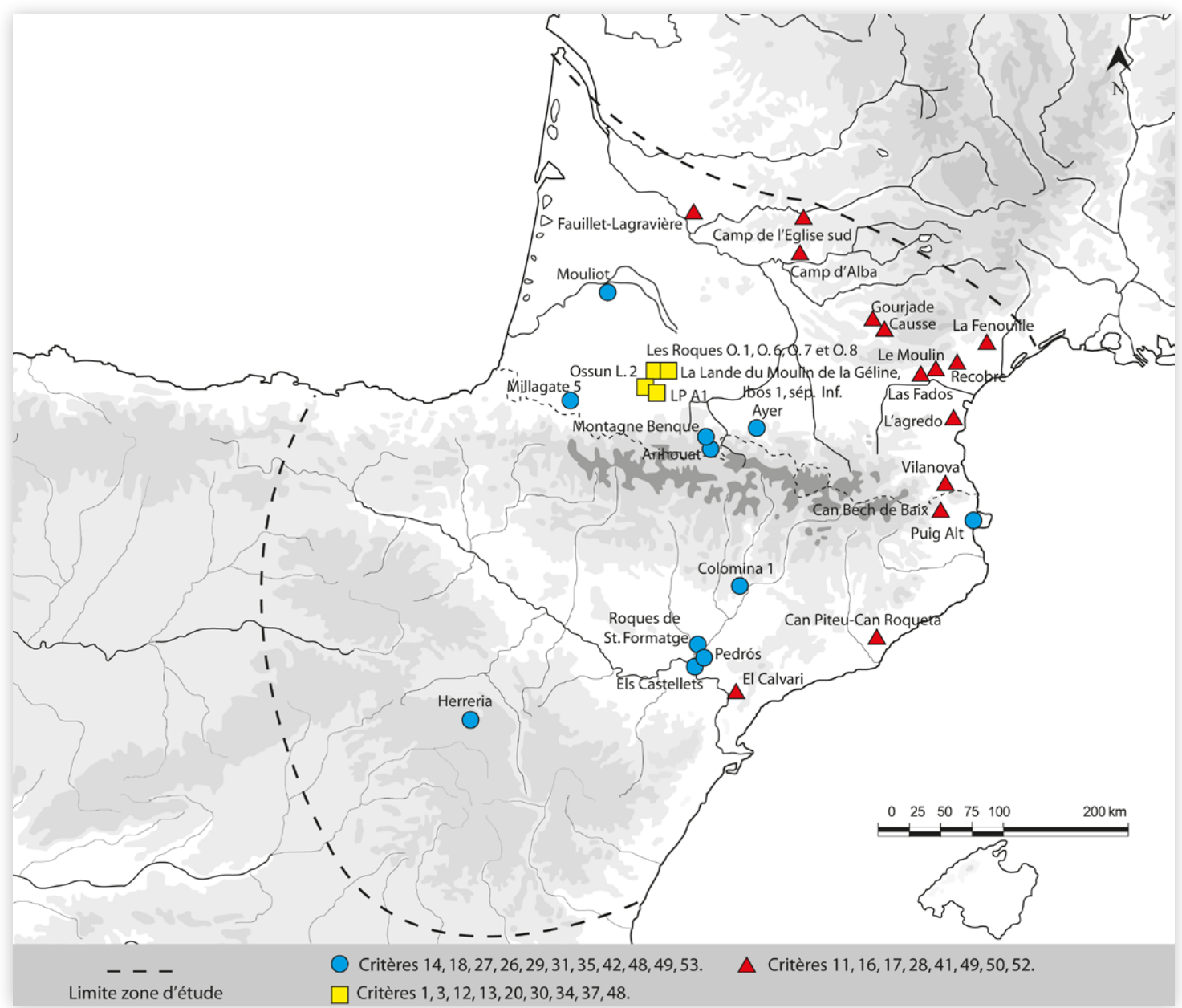

Figure 4 :

carte de répartition des " séquences funéraires » des principales nécropoles de l'étape I (S. Adroit)

\section{Groupe funéraire 3}

Le troisième groupe comprend principalement des nécropoles où le dépôt funéraire est déposé dans un loculus de plan circulaire [18]. Parfois, des témoins de dispositifs de fermeture en pierres de la fosse sont conservés [19]. Les nécropoles à crémation de ce groupe ont aussi livré des restes de constructions qui se présentent sous la forme de micro-tumulus. Nous observons également l'existence de structures périphériques d'entourage autour des loculi. Ces structures délimitent la surface occupée en son centre par la fosse sépulcrale et permettent aussi de circonscrire le remplissage de terre et/ou de pierres [20]. Cependant, les entourages reconnus sur ces nécropoles sont de plusieurs types : cercles de dalles plantées de chant, cercles de pierres posées à même le sol, etc. Pour ce qui est de l'organisation générale de ces nécropoles, trois constantes les dissocient des deux groupes précédents. Tout d'abord, elles se trouvent préférentiellement au niveau des plaines et des terrasses alluviales. Ensuite, il s'agit de nécropoles regroupant un nombre de sépultures largement supérieur aux ensembles sépulcraux des deux premiers groupes. Enfin, quelques-unes des nécropoles de ce groupe montrent de nombreux exemples de superposition de tombes, ce qui prouve qu'elles n'étaient pas signalées de façon pérenne.
Maintenant, si I'on observe la répartition géographique de ces différents groupes nous nous apercevons que le premier se trouve en Aquitaine méridionale, c'est-à-dire dans la zone du plateau de Ger, le deuxième se situe dans les Pyrénées centrales ainsi qu'au niveau de la confluence des rivières du Sègre et du Cinca, enfin le troisième groupe, beaucoup plus lâche, se concentre sur le littoral catalano-languedocien et le long de l'axe AudeGaronne. Les différents groupes se répartissent de façon plus ou moins homogène dans l'espace. Ces groupes funéraires recoupent en partie seulement les groupes culturels du Sègre-Cinca, du Languedoc occidental et de I'Aquitaine méridionale définis par les archéologues.

\section{Étape II : le premier âge du Fer ancien}

Pour le début du premier âge du Fer, nous avons suivi la même méthode d'analyse à partir de la sélection de 33 nécropoles à crémation répertoriées dans le cadre de l'étude (Fig. 5).

[18] Vilanova à Céret (Pyrénées-Orientales), Recobre à Quarante (Hérault), Las Fados à Pépieux (Hérault).

[19] Moulin à Mailhac (Aude), Can Bech de Baix à Agullana (Alt Empordà, Girona), Can Piteu-Can Roqueta à Sabadell (Vallès occidental, Barcelona).

[20] Camp de I'Église Sud à Flaujac-Poujols (Lot). 


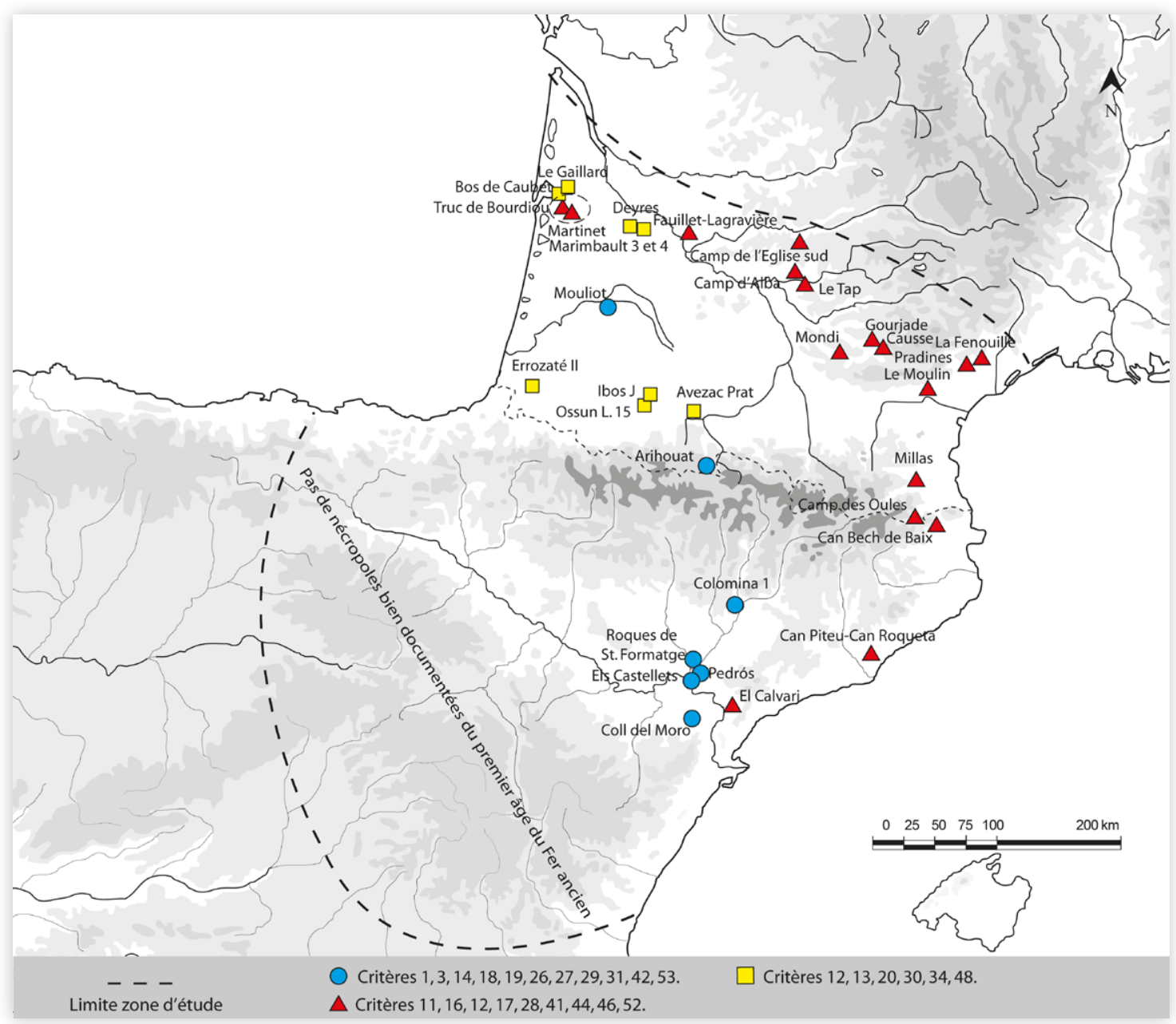

Figure 5 :

carte de répartition des « séquences funéraires » des principales nécropoles de l'étape II (S. Adroit)

\section{Groupe funéraire 1}

Le premier groupe s'individualise par des tertres de grandes dimensions, des cercles de galets concentriques et des sépultures adventices dans la masse tumulaire. Comme à l'étape I, la fosse sépulcrale est souvent entourée d'un cercle de galets bordant le pourtour du tumulus. Dans quelques cas, avant l'érection du tumulus, la surface destinée à son aménagement est creusée. Ces nécropoles comptabilisent un nombre de sépultures relativement limité. Pour ce qui est de l'organisation générale des nécropoles, nous observons une disposition aléatoire des tertres. Cependant, il existe des nécropoles qui ont livré des plans en forme de trèfle, comme les nécropoles du Bos de Caubet et de la Houn de la Peyre à Biganos (Gironde) [21].

\section{Groupe funéraire 2}

Le deuxième groupe identifié est également récurrent puisqu'il avait déjà été défini à l'étape antérieure (zones des Pyrénées et du Sègre et du Cinca). Il se définit par des dépôts funéraires placés dans un loculus ou à l'intérieur d'une ciste en pierre au centre du monument funéraire. Le tout est ceinturé par une couronne étroite de pierres qui délimite une surface de faible diamètre.

\section{Groupe funéraire 3}

Le troisième groupe comprend des nécropoles à crémation qui se composent majoritairement de dépôts de crémation secondaire placés à l'intérieur d'un vase. Par la suite, le vase contenant les restes humains calcinés est déposé dans une fosse de forme cylindrique ou parfois de forme parallélépipédique. Par ailleurs, nous avons répertorié plusieurs cas de restes humains calcinés mêlés à un sédiment charbonneux. Ces résidus de crémation se trouvent dans le comblement de la fosse sépulcrale ou sur la couverture des sépultures comme dans la nécropole du Moulin à Mailhac (Aude) [22]. La fosse sépulcrale est ensuite condamnée, soit simplement par une (ou plusieurs) dalle(s), soit par un système qui associe une armature en bois et une dalle de pierre. Ainsi, sur ces dalles de couverture, nous retrouvons plusieurs dispositifs de signalisation, comme un petit tumulus (entre 1 et $4 \mathrm{~m}$ de diamètre) de pierres ou de terre, des structures périphériques d'entourage autour des loculi, etc.

[21] Mohen \& COFFYn 1970, p. 13-14 et p. 29-31.

[22] TAFFAnEL et al. 1998. 


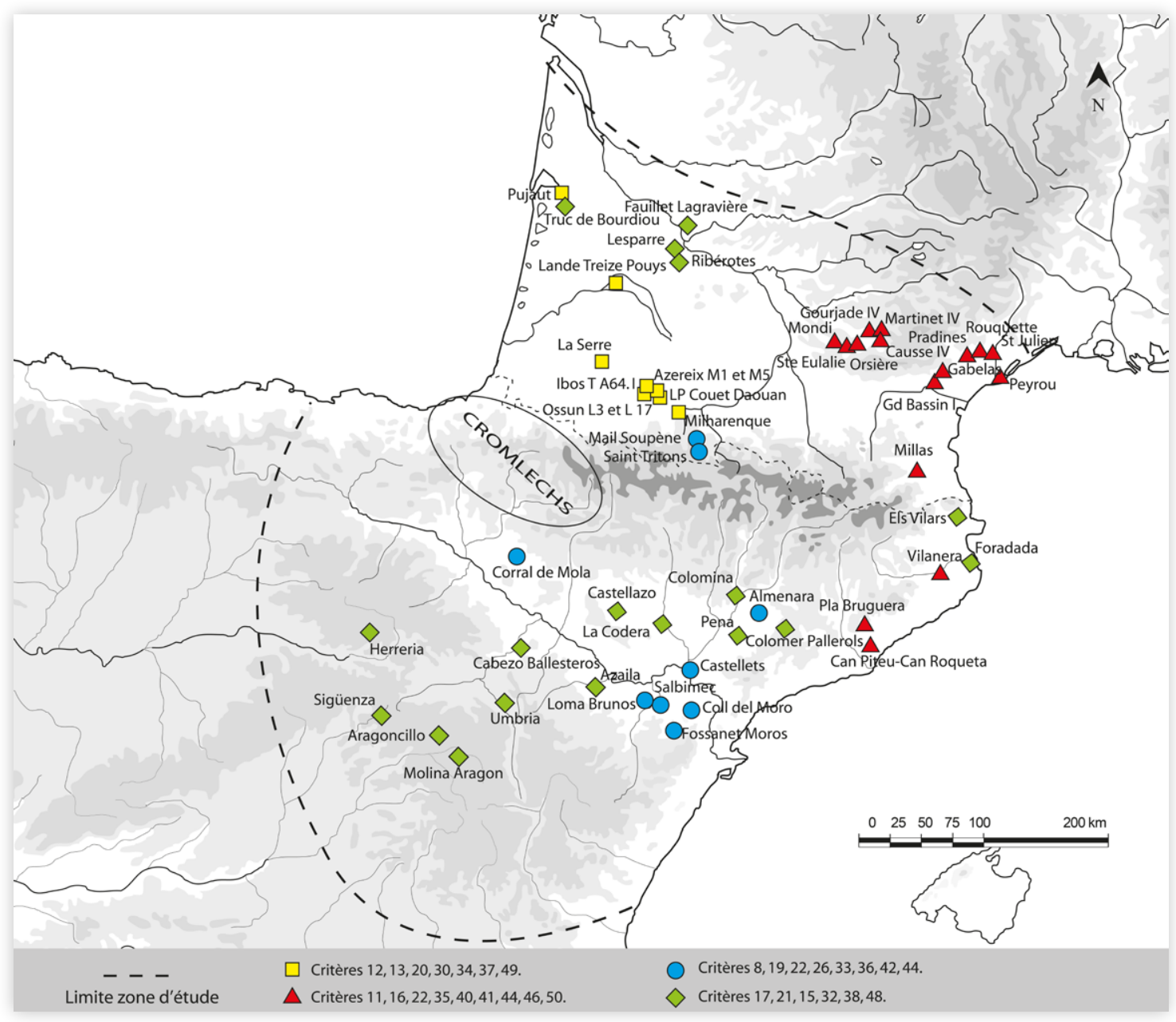

Figure 6 :

carte de répartition des « séquences funéraires " des principales nécropoles de l'étape III (S. Adroit)
Concernant l'organisation générale de ces nécropoles, nous remarquons qu'il s'agit souvent d'ensembles avec des effectifs atteignant facilement la centaine de tombes. La carte de répartition signale des groupes funéraires ancrés de manière homogène sur de vastes espaces géographiques. La continuité des séquences funéraires avec I'étape II démontre une certaine stabilité des pratiques funéraires. En revanche, nous pensons que l'absence d'homogénéité observée pour le nord du département aquitain est en partie imputable à la conservation différentielle des nécropoles.

\section{Étape III : les nécropoles du premier âge du Fer moyen}

Pour la phase moyenne du premier âge du Fer nous avons sélectionné 54 nécropoles à crémation sur le territoire étudié (Fig. 6).

\section{Groupe funéraire 1}

Le premier groupe se concentre sur un espace géographique cohérent et quasiment identique à celui du groupe funéraire 1 de l'étape II. Il s'individualise par des tertres monumentaux qui ont un périlithe de galets, d'un diamètre compris entre 10 et $20 \mathrm{~m}$. De surcroît, si durant les étapes antérieures il n'y avait en général qu'une seule sépulture dans les tumulus, à partir du premier âge du Fer moyen, les tumulus deviennent collectifs formant de véritables nécropoles communautaires [23]. Pour illustrer cette idée nous pouvons prendre l'exemple du tumulus $A$. 64. 1 d'Ibos (plateau de Ger, Hautes-Pyrénées) qui a livré une soixantaine de dépôts de crémation secondaire qui $s^{\prime}$ échelonnent entre le $v^{\mathrm{e}}{ }^{\mathrm{e}}$ et le milieu du Ive s. av. J.-C. [24]. Les tumulus de Milharenque, de la nécropole de la Coustalade à Avezac-Prat-Lahitte (Hautes-Pyrénées) ont aussi livré des restes de bûcher infra-tumulaire [25].

\section{Groupe funéraire 2}

Un deuxième groupe s'individualise par des types funéraires bien particuliers. Finalement, nous écarterons les deux nécropoles du domaine pyrénéen central car il s'agit de découvertes anciennes, ce qui pourrait biaiser notre analyse. Les nécropoles de la région du Bas-Aragon sont conglomérées sur le graphique (El Salbimec, Fossanet del Moros, La Loma de los Brunos).

[23] EsCudÉ-Quillet 1998.

[24] EsCudÉ-Quillet 2007.

[25] EsCudÉ-Quillet 2002. 
Traditionnellement dans la littérature archéologique les tumulus de la région du Bas-Aragon sont regroupés sous I'appellation générique de tumulus à ciste excentrée et constituent un groupe funéraire bien défini par les archéologues [26]. La majorité des aires funéraires du Bas-Aragon se compose d'un faible nombre de tombes. Pour ce qui est de l'architecture funéraire, la plupart de ces tombes correspondent à des structures tumulaires de plan circulaire à ciste excentrée en dalle de pierre. Ces tumulus ont des diamètres compris entre 3 et $5 \mathrm{~m}$. Quelques-uns des tumulus fouillés dans les nécropoles du Coll del Moro à Gandesa (Terra Alta, Tarragona) et de La Loma de Los Brunos à Caspe (Bajo-Aragón-Caspe, Zaragoza) ont une ciste subsidiaire adossée au tumulus qui servait peut-être à une sépulture annexe ou au dépôt d'une offrande alimentaire [27] .

\section{Groupe funéraire 3}

Le troisième groupe se définit par des micro-tumulus comprenant le plus souvent une structure d'entourage étroite faite de pierres ou d'adobes. Les loculi qui accueillent le dépôt funéraire sont fréquemment comblés avec les résidus de la crémation provenant du bûcher funéraire. Pour ce qui est des dispositifs de signalisation, il en existe de plusieurs types : amas pierreux en vrac, dallage, etc.

\section{Groupe funéraire 4}

Le quatrième groupe comprend des nécropoles de grande envergure dont beaucoup d'entre elles ont bénéficié de fouilles récentes. Les sépultures de ces ensembles funéraires se présentent sous la forme de micro-tumulus de plan quadrangulaire ou circulaire. Les fosses des sépultures en question adoptent des formes cylindriques, sub-circulaires, en silo ou parallélépipédiques [28]. Les tombes étaient fermées par une ou plusieurs dalles puis signalées par un micro-tumulus. Les sépultures des nécropoles du Castrais s'inscrivent dans la continuité par rapport aux étapes antérieures. Nous mentionnerons seulement l'apparition de sépultures annexes à simple ossuaire comme dans les nécropoles du Bas-Languedoc. Le dépôt de faune (quartiers de viande) dans le loculus est aussi I'un des traits récurrent de ce groupe funéraire.

La carte de répartition de ces nécropoles paraît montrer une régionalisation des pratiques funéraires plus marquée qu'aux étapes antérieures. Toutefois, nous

[26] Pour une synthèse de la question, voir RAFEL 2003.

[27] RAFEL 2003.

[28] Grand Bassin I à Mailhac (Aude), Can Piteu-Can Roqueta à Sabadell (Vallès Occidental, Barcelona), Vilanera à L'Escala (Alt Empordà, Girona). observons que cette régionalisation accrue n'est pas totalement homogène. En effet, la séquence funéraire 4 se répartit sur une surface importante et discontinue puisque son aire de répartition est partagée par d'autres séquences funéraires.

\section{ÉTAPE IV : les nécropoles du premier âge du Fer récent}

L'analyse factorielle de la dernière étape du premier âge du Fer montre que les nécropoles se regroupent essentiellement vers le centre du graphique. Toutefois, il est possible d'observer grosso modo trois groupes (Fig. 7).

\section{Groupe funéraire 1}

Le premier groupe s'individualise par des tertres en terre de grandes dimensions. Les tumulus possèdent les mêmes critères typologiques que le groupe 1 du premier âge du Fer moyen qui se trouve également en Aquitaine. En effet, il s'agit de tumulus communautaires qui concentrent un grand nombre de sépultures et dont les pratiques funéraires n'évoluent pas par rapport aux étapes chronologiques antérieures.

\section{Groupe funéraire 2}

Le deuxième groupe se compose de sépultures en fosse dans laquelle les restes humains du défunt sont déposés, soit à l'intérieur d'un vase cinéraire, soit sans aucun contenant. Les fosses de ces sépultures sont quelquefois composées d'un surcreusement au fond de la fosse destiné à caler le vase cinéraire. Elles sont ensuite, le plus souvent, comblées avec les résidus de la crémation : les débris de terre cuite, les restes osseux brûlés, les objets métalliques et les tessons. Les archéologues retrouvent fréquemment dans le remblai de ces fosses des tessons non brûlés et des tessons brûlés. Ces traces de chauffe prouvent -au même titre que le mobilier métallique fondu- que quelques vases étaient placés sur le bûcher funéraire avec le corps du défunt. Lorsque les nécropoles n'ont pas été totalement arasées, il y a parfois des traces d'un amas de pierres surmontant la fosse ou des dalles en calcaire qui ceinturent la fosse à la manière d'un petit tumulus.

\section{Groupe funéraire 3}

Le troisième groupe se définit par des nécropoles qui ont livré majoritairement des structures tumulaires même si nous observons une grande variabilité de types et de matériaux. Il s'agit la plupart du temps de tumulus circulaires constitués d'un parement étroit de pierres ou d'adobes. Cette structure d'entourage peut être faite de dalles plantées de chant qui atteignent parfois $70 \mathrm{~cm}$ de haut, comme dans la nécropole d'Arroyo Vizcarra à Urriés 


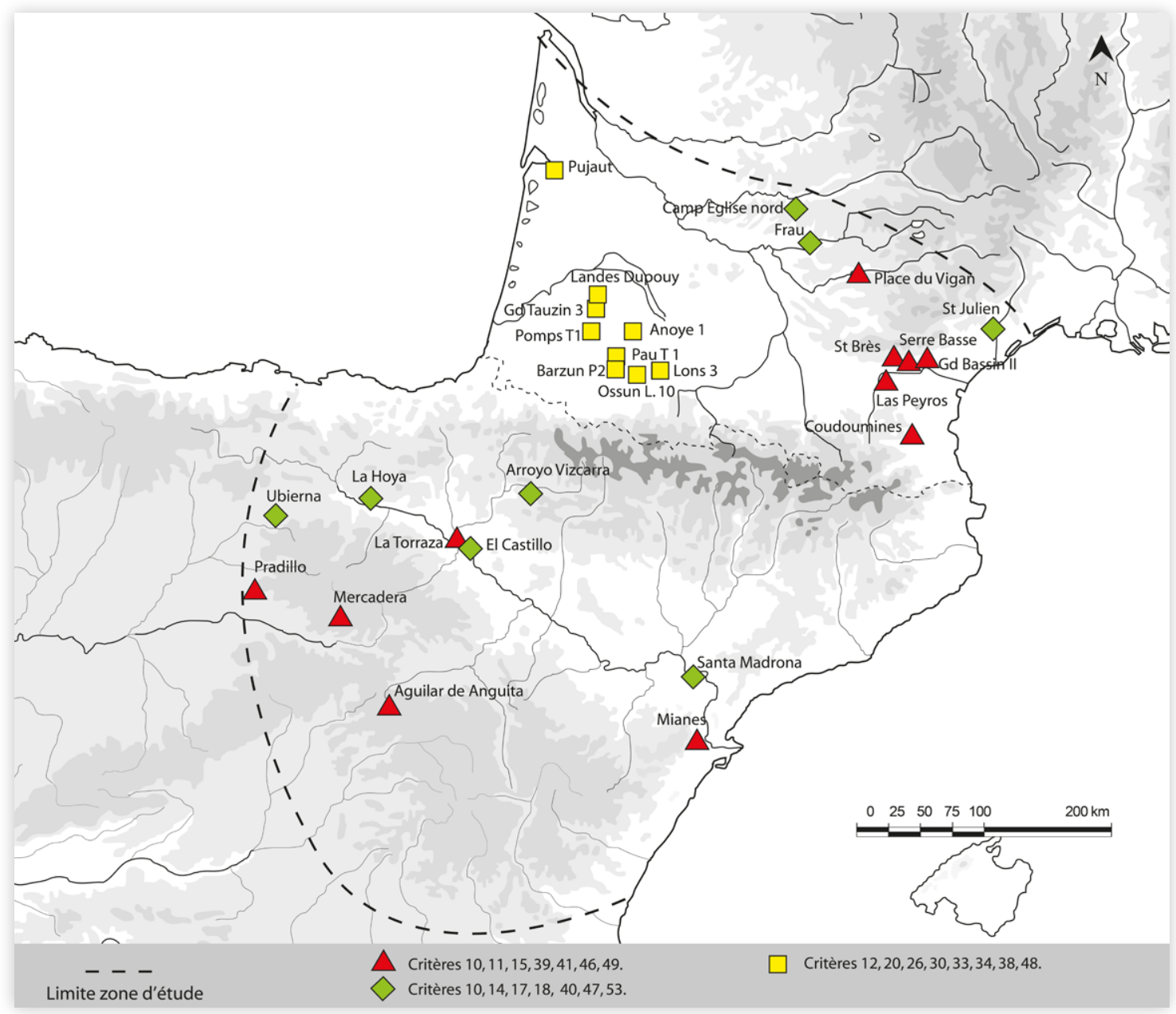

Fig. 7 :

carte de répartition des « séquences funéraires » des principales nécropoles de l'étape IV (S. Adroit)
(Zaragoza) [29], ou d'adobes superposées sur au moins cinq assises dans la nécropole d'El Castillo à Castejón (Navarra) [30]. Les dimensions des tumulus sont extrêmement variables à l'intérieur de ce groupe funéraire, mais également intra-site. Par exemple, les nécropoles du Frau à Cazals (Tarn-et-Garonne) et du Camp de l'Église Nord à Flaujac-Poujols (Lot) montrent des tumulus de grandes dimensions (entre 28 et $44 \mathrm{~m}^{2}$ de superficie en moyenne dans ces nécropoles respectives) alors que les nécropoles de Santa Madrona à Riba-Roja d'Ebre (Ribera d'Ebre, Tarragona) et de la Polera à Ubierna (Burgos) ont révélé des tumulus de petite taille [31]. De surcroît, plusieurs de ces tumulus étaient signalés par des stèles en pierre occasionnellement décorées (ex : Aguilar de Anguita, La Hoya). Ce groupe comprend également des nécropoles qui ont livré des indices de crémation primaire comme les nécropoles d’Arroyo Vizcarra à Urriés (Zaragoza) et d'El Castillo à Castejón (Navarra). Les restes humains des crémations secondaires sont introduits dans un contenant céramique ou ils sont simplement déposés à l'intérieur de la structure sépulcrale sans aucune protection particulière.

[29] Royo GuilLÉn 1997.

[30] Faro Carballa \& Unzu Urmeneta 2006.

[31] Pajot 1984 ; Beausoleil et al. 2007 ; Belarte \& Noguera 2007 ; RuIz Vélez 2001.
Le dépôt funéraire peut être mis directement à la surface du sol, à l'intérieur d'une fosse ou d'une ciste en pierre. Contrairement à la plupart des nécropoles du groupe 2, les tombes ont livré des dépôts alimentaires carnés.

La carte de répartition montre qu'il existe des « séquences funéraires » qui appartiennent à un espace géographique en particulier (c'est le cas notamment du groupe 1). Néanmoins on s'aperçoit, d'ailleurs comme dans les autres étapes chronologiques, qu'il existe parfois une grande variabilité des pratiques funéraires au sein d'un espace géographique restreint.

\section{EN GUISE DE CONCLUSION}

Au premier abord, plusieurs traits funéraires semblent clairement répondre à des normes et à des usages fixés par la communauté, voire au-delà dans un réseau plus vaste de relations avec d'autres communautés. Pour preuve nous mentionnerons le fait que parmi les critères que nous avons définis quelques-uns sont adoptés presque sur l'intégralité de l'espace étudié et sont invariants aussi bien spatialement que chronologiquement (sépultures individuelles en vase, sous-représentation des jeunes enfants dans les nécropoles, etc.). 
À l'inverse de ces dynamiques inter-régionales communes, d'autres pratiques funéraires sont propres à certaines contrées. Nous observons sur les cartes de répartition des groupes funéraires qui occupent des espaces géographiques cohérents et souvent homogènes, quelle que soit l'étape concernée (ex : groupe funéraire 1 en Aquitaine). Par ailleurs, les cartes de répartition montrent également des dynamiques funéraires similaires sur le pourtour du littoral méditerranéen, tant au niveau des pratiques que dans le type de mobilier usité. Des affinités culturelles remarquées, essentiellement sur la base du mobilier, entre certains groupes culturels semblent également existées dans les pratiques funéraires.
Par exemple, le groupe du Languedoc occidental et celui du Tarn montrent des pratiques funéraires semblables au cours des étapes étudiées. À partir du premier âge du Fer moyen on observe que la nécropole d'Els Castellets à Mequinenza (Zaragoza) est associée aux nécropoles tumulaires du Bas-Aragon ce qui vient conforter les hypothèses de quelques chercheurs espagnols comme G. Ruiz Zapatero lorsqu'il évoque les relations existant entre le «groupe du Sègre-Cinca » et le «groupe du Bas-Aragon ». Cette étude comparative des nécropoles à crémation de la première moitié du Ier millénaire av. J.-C. nous permettra de questionner à nouveaux frais les groupes culturels identifiés par nos prédécesseurs.

BaraY, Luc, 2003, Pratiques funéraires et sociétés de l'âge du Fer dans le bassin Parisien (fin du vir ${ }^{e}$ s. - troisième quart du $I I^{e}$ S. avant J.-C.) (Gallia suppl. 56), Paris.

Beausoleit, Jean-Michel et al., 2007, «La nécropole protohistorique (VI - ve s. a.C.) du Camp de l'Église Nord (FlaujacPoujols, Lot). Approche préliminaire et premiers résultats», dans Michel Vaginay \& Lionel Izac-Imbert (éd.), Les âges du Fer dans le sud-ouest de la France, XXVIII e colloque de l'AFEAF à Toulouse, 2004 (Aquitania, suppl. 14, vol. 1), p. 125-151.

Belarte, Maria Carme \& Noguera, Jaume, 2007, La Necròpolis Protohistòrica de Santa Madrona (Riba-Roja d'Ebre, Ribera $d^{\prime} E b r e$ ), Institut Català d'Arqueologia Clàssica (Hic et nunc 2), Tarragona.

Bosch Gimpera, Pere, 1932, Etnología de la Península Ibérica, Jordi Cortadella (éd.) (2003), Pamplona.

Cerdeño, María Luisa, 1978, «Los broches de cinturón peninsulares de tipo céltico », Trabajos de Prehistoria 35, p. $279-306$.

Cerdeño, María Luisa \& Chordá, Marta, 2004, « Fíbulas de tipo navarro-aquitano en el área celtibérica », Cuadernos de Arqueología de la Universidad de Navarra 12, p. 161-175.

DudAY, Henri et al., 2000, « Validation des paramètres de quantification, protocoles et stratégies dans l'étude anthropologique des sépultures secondaires à incinération. L'exemple des nécropoles protohistoriques du Midi de la France », dans Bernard Dedet, Philippe Gruat, Georges Marchand, Michel Py \& Martine Schwaller (éd.), Archéologie de la mort, Archéologie de la tombe au premier âge du Fer, Actes du colloque de Conques-Montrozier 1997 (Monographies d'Archéologie Méditerranéenne 5), Lattes, p. 7-30.

Escudé-Quillet, Jean-Marie, 1998, Du complexe pyrénéen au complexe sud-aquitain la fin de l'âge du Bronze et l'âge du Fer de l'Aquitaine méridionale, Thèse doctorale, Université de Toulouse II-Le Mirail.

Escudé-Quillet, Jean-Marie, 2002, « Fouille des tumulus de Milharenque, Nécropole de la Coustalade (VII ${ }^{\mathrm{e}}$ siècle avant notre ère). Avezac-Prat-Lahitte, Hautes-Pyrénées », Archéologie des Pyrénées occidentales et des Landes 21, p. 127-150.

Escudé-Quillet, Jean-Marie, 2007, « Pour une chronologie de l'âge du Fer de l'Aquitaine méridionale (VI ${ }^{\mathrm{e}-\mathrm{IV}} \mathrm{e} \mathrm{S}$. a. C.) : étude chronotopographique du tumulus A. 64. 1 (Ibos, Hautes-Pyrénées) », dans Michel Vaginay \& Lionel Izac-Imbert (éd.), Les âges du Fer dans le sud-ouest de la France, xxvIII colloque de l'AFEAF à Toulouse, 2004 (Aquitania, suppl. 14, vol. 1), p. 93-123.

Faro Carballa, José Antonio \& Unzu Urmeneta, Mercedes, 2006, « La necrópolis de la Edad del Hierro de El Castillo (Castejón, Navarra). Primeras valoraciones: campañas 2000-2002 », Complutum 17, p. 145-166.

GaIlledrat, Éric, 1997, Les Ibères de l'Ėbre à I'Hérault : VI ${ }^{e}-I V^{e}$ s. avant J.-C. (Monographies d'Archéologie Méditerranéenne 1), Lattes.

Garcia, Dominique, 2004, La Celtique méditerranéenne. Habitats et sociétés en Languedoc et en Provence du viII au II siècle av. J.-C. (Collection des Hespérides), Paris.

Graells, Raimon, 2008, La necròpolis protohistòrica de Milmanda (Vimbodí, Conca De Barberà, Tarragona): un exemple del món funerari català durant el trànsit entre els segles viII - VI aC, Institut Català d'Arqueologia Clàssica (Hic et nunc 5), Tarragona.

JANIN, Thierry, 1994, «Systèmes de couverture et dispositifs de signalisation reconnus dans la nécropole du Moulin à Mailhac (Aude) », Documents d'Archéologie Méridionale 17, Lattes - Montpellier, p. 39-46. 
López Cachero, Javier, 2005, La necrópolis de Can Piteu-Can Roqueta (Sabadell) en el contexto del Bronce final y la primera edad del Hierro en el Vallès: estudio de los materiales cerámicos, Tesis doctoral, Universitat de Barcelona.

López Cachero, Javier \& Rovira i HortalÀ, Carme, 2012, «El món funerari a la depressió prelitoral catalana entre el bronze final i la primera edat del ferro : ritual i dinamisme social a partir del registre arqueològic » dans Carme Rovira i Hortalà, Javier López Cachero \& Florent Mazière (éd.), Les necròpolis d'incineració entre l'Ebre i el Tíber (segles IX-VI aC) : metodologia, pràctiques funeràries i societat (Monografies Museu d'Arqueologia de Catalunya 14), p. 37-55.

Louis, Maurice et al., 1960, Le premier âge du Fer languedocien. Les tumulus, conclusions (Collection de monographies préhistoriques et archéologiques 3, III), Bordighera.

LucE, Jean-Marc, 2007, « Géographie funéraire et identités ethniques à l'âge du Fer en Grèce », dans Jean-Marc Luce (éd.), Identités ethniques dans le monde grec antique, Actes du colloque international du CRATA de Toulouse, 2006, Pallas 73, p. 39-51.

Mohen, Jean-Pierre, 1980, L'âge du Fer en Aquitaine du VIII ${ }^{e}$ au III siècle avant J.-C. (Mémoires de la Société Préhistorique Française 14), Paris.

Mohen, Jean-Pierre \& Coffyn, André, 1970, Les nécropoles hallstattiennes de la région d'Arcachon (Bibliotheca Praehistorica Hispana XI), Madrid.

Pajot, Bernard, 1984, « Architecture des tumulus à incinération de la nécropole du Frau (Cazals, Tarn-et-Garonne) 》, Antiquités Nationales 16/17, p. 97-117.

Rafel, Núria, 2003, Les Necròpolis tumulàries de tipus baixaragonès: les campanyes de l'Institut d'Estudis Catalans al Matarranya, Institut d'Estudis Catalans et Museu d'Arqueologia de Catalunya (Monografies del MAC 4), Barcelona.

Royo Guillen, José Ignacio, 1997, «La necrópolis de incineración del Arroyo Vizcarra en Ruesta (Urriés, Zaragoza) », Arqueología Aragonesa 1993, p. 47-58.

Royo Guillen, José Ignacio, 2000, «Tipología funeraria, ritos y ofrendas en las necrópolis del valle del Ebro durante la Primera Edad del Hierro (s. viII-s.v a.C.) », dans Bernard Dedet, Philippe Gruat, Georges Marchand, Michel Py \& Martine Schwaller (éd.), Archéologie de la mort, Archéologie de la tombe au premier âge du Fer, Actes du colloque de Conques-Montrozier 1997 (Monographies d'Archéologie Méditerranéenne 5), Lattes, p. 41-58.

Ruiz VéLez, Ignacio, 2001, El ritual funerario en las necrópolis burgalesas de la edad del hierro, Institución Fernán González Academia Burgense de Historia y Bellas Artes, Burgos.

Ruiz Zapatero, Gonzalo, 2004, « Casas y tumbas. Explorando la desigualdad social en el bronce Final y primera edad del Hierro del NE de la Península Ibérica », Mainake 26, p. 293-330.

TAffanel, Odette et al., 1998, La nécropole du Moulin à Mailhac (Aude) (Monographies d'Archéologie Méditerranéenne 2), Lattes. 